\title{
Fever of Unknown Origin in the Elderly: Lymphoma Presenting as Vertebral Compression Fractures
}

\author{
Kimberly Y. Smith, Suzanne F. Bradley, and Carol A. Kauffman
}

$\mathrm{I}^{\mathrm{n}}$ n elderly patients, the syndrome of fever of unknown origin (FUO) differs in several important regards from that seen in younger patients. The causes of FUO in the elderly are rarely benign or selflimited; almost always, an elderly patient with FUO will be found to have a serious illness as the cause of the fever. ${ }^{1-6}$ Factitious and fraudulent causes of FUO are exceedingly rare in the elderly. 7,8

We present the cases of two elderly men with FUO, which was ultimately shown to be caused by lymphoma. Both had back pain with vertebral compression fractures related to the neoplasms, and initially both were presumed to have these fractures secondary to osteoporosis. This presumption contributed to a delay in diagnosis of the cause of the FUO in both patients.

\section{CASE REPORTS}

Case 1 A 63-year-old man presented in October 1991 with a 4-week history of chills, fevers, back and abdominal pain, and a 17 -pound weight loss. His past medical history was significant for chronic obstructive pulmonary disease, peptic ulcer disease with subtotal gastrectomy, and hematuria. He had a 100-pack-year smoking history and a history of alcohol abuse.

Physical examination revealed a thin, elderly, illappearing man with a respiratory rate of 32 breaths per minute, a temperature of $100.2^{\circ} \mathrm{F}\left(37.9^{\circ} \mathrm{C}\right)$, blood pressure of $110 / 70 \mathrm{mmHg}$, and pulse of 104 beats per minute. The only abnormalities on physical examination were decreased breath sounds, a diffusely tender abdomen, and tenderness over the thoracic spine.

The white blood cell count was $10,000 / \mathrm{ml}$ with $68 \%$ neutrophils, $20 \%$ lymphocytes, $11 \%$ monocytes, and $1 \%$ eosinophils; hematocrit was $25 \%$, and platelet count was $177,000 / \mathrm{ml}$. Westergren erythrocyte sedimentation rate (WESR) was $123 \mathrm{~mm} /$ hour. The aspartate aminotransferase was $85 \mathrm{IU} / \mathrm{L}$, alanine aminotransferase was $18 \mathrm{IU} / \mathrm{L}$, and total bilirubin was 1.3 $\mathrm{mg} / \mathrm{dL}$. Urinalysis revealed large amounts of blood.

Chest roentgenogram showed no infiltrates but did show a T9 compression fracture, which was felt initially to be secondary to osteoporosis (Figure 1). Computed tomographic (CT) scan showed a T9 vertebral fracture that did not suggest neoplasm or osteomyelitis. A bone scan showed no uptake in the spine. In spite of these negative findings and because of the history of fever,

From the Divisions of Infectious Diseases and Geriatrics, Department of Internal Medicine, Department of Veterans Affairs Medical Center, University of Michigan Medical Center, Ann Arbor, Michigan.

Address correspondence and reprint requests to Dr. Carol A. Kauffman Veterans Affairs Medical Center, 2215 Fuller Road, Ann Arbor, MI 48105. vertebral compression fracture, and elevated sedimentation rate, a presumptive diagnosis of vertebral osteomyelitis was made. Multiple cultures of blood yielded no growth. The patient was started on vancomycin and ampicillin/sulbactam. In spite of antibiotic therapy, the patient continued to have fevers as high as $104.2^{\circ} \mathrm{F}$ $\left(40.1^{\circ} \mathrm{C}\right)$.

Because of a poor response to antimicrobial therapy, persistently elevated WESR ( $86 \mathrm{~mm} /$ hour), weight loss, and anemia, the possibility of malignancy was raised. Esophagogastroduodenoscopy and colonoscopy were normal, intravenous pyelogram showed incomplete obstruction at the left ureteropelvic junction, and retrograde pyelogram revealed an extrarenal pelvis with ureteropelvic obstruction. Bone marrow biopsy revealed an increase in the granulocytic series and plasma cells; cultures yielded no growth. CT scan revealed inflammatory changes in the pancreas, two lytic lesions in the liver, and an enlarged retrocrural lymph node. Ultrasound-guided fine-needle biopsy of one of the liver lesions revealed necrotic cells and macrophages, and culture yielded no growth. It was felt that he might have a liver abscess, and antibiotics were continued.

Serologic studies for fungal, viral, and bacterial pathogens were nonrevealing. He was anergic to both tuberculin and Candida antigens. Antinuclear antibodies, rheumatoid factor, and complement studies were negative or within normal limits.

After 5 weeks of fever, while still on antibiotics, the patient developed increasing weakness, shortness of breath, and confusion. Bilateral lower extremity weakness and clonus appeared. Magnetic resonance (MR) imaging showed destruction of $\mathrm{T} 9$, a paravertebral mass anterior to the vertebral body, and cord compression (Figure 2). Needle biopsy of the paravertebral mass was performed but was nondiagnostic. Over the next few days he became progressively more disoriented and died approximately 3 months after the illness began.

Necropsy revealed large-cell lymphoma involving pancreas, peripancreatic and perinephric lymph nodes, heart, liver, and T9 vertebral body. Although examination of the brain was not permitted, cytologic examination of cerebrospinal fluid revealed abnormal lymphocytes typical of meningeal lymphoma.

Case 2 A 74-year-old man presented in February 1991 with a 3-month history of intermittent fevers to $102^{\circ} \mathrm{F}\left(38.9^{\circ} \mathrm{C}\right)$, weakness, a 20 -pound weight loss, and myalgias. Extensive work-up at another hospital had revealed no cause for his fevers. His past medical history was significant for pneumonia, nephrolithiasis, and prostatitis. The patient was a retired jockey; he 


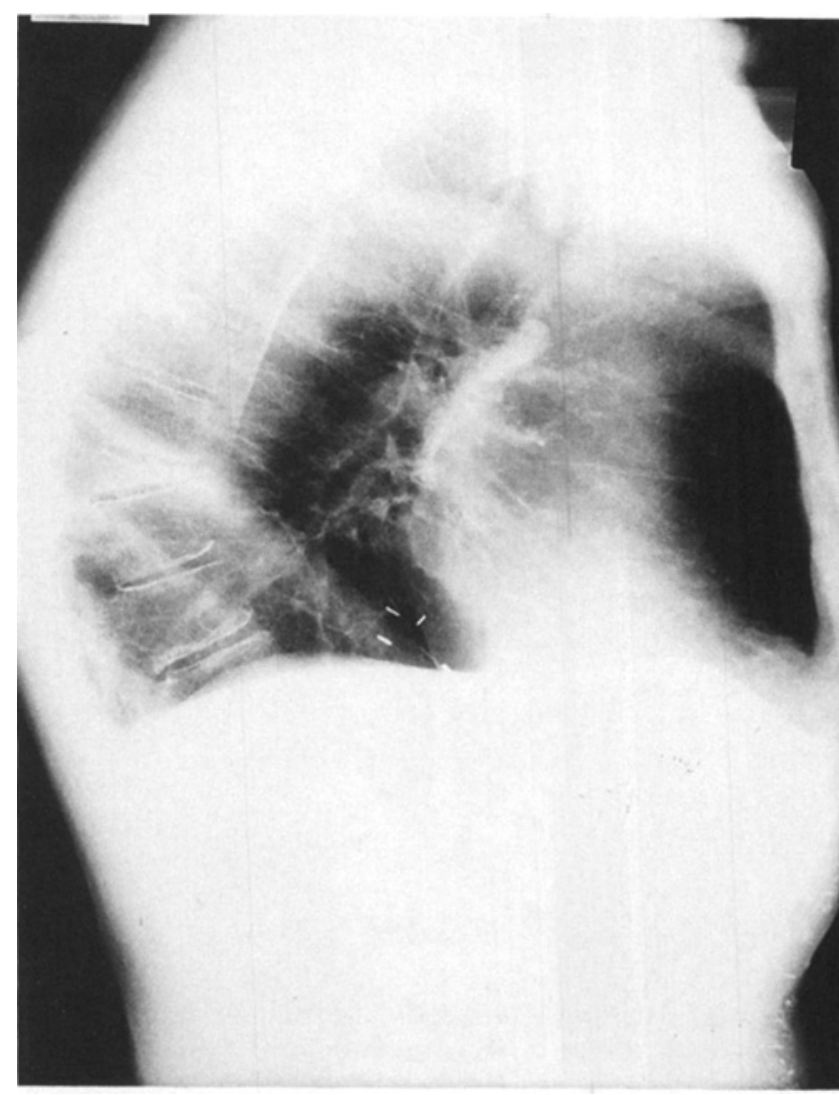

FIGURE 1. Patient 1. Lateral chest roentgenogram showing $T 9$ compression fracture without narrowing of intervertebral disk spaces or disruption of the end plates of the vertebral bodies.

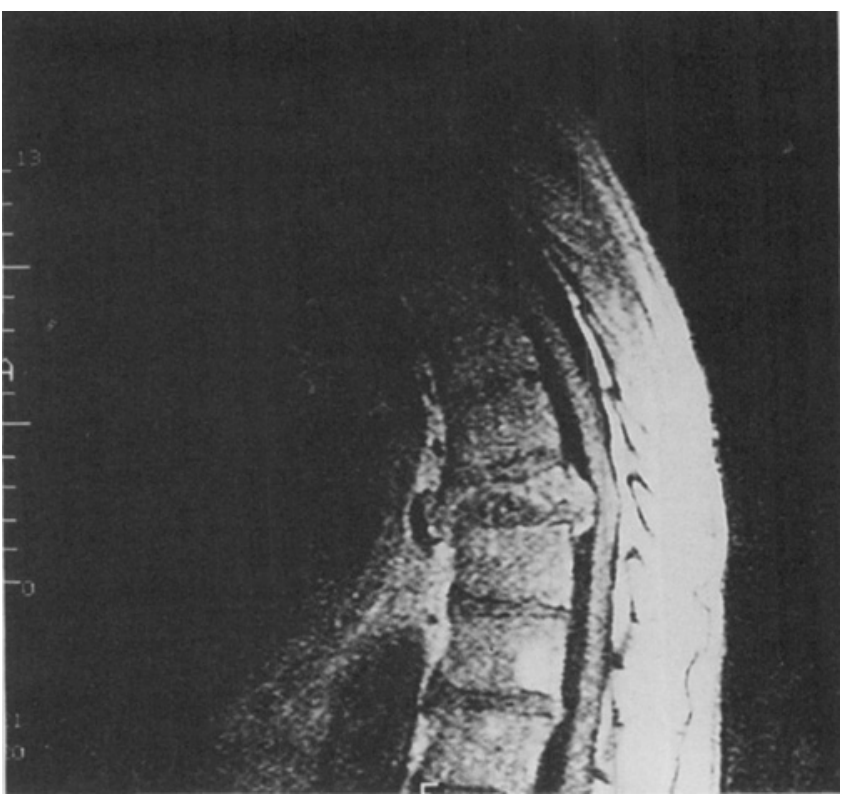

FIGURE 2. Patient 1. MR imaging study with gadolinium enhancement showing destruction of the T9 vertebral body with extension of the process anteriorly, impingement on the spinal cord posteriorly, and preservation of the intervertebral disk spaces.

had a 40-pack-year smoking history and did not drink alcohol.

Physical examination revealed a frail-appearing, elderly man with a temperature of $99^{\circ} \mathrm{F}\left(37.2^{\circ} \mathrm{C}\right)$, blood pressure $142 / 67 \mathrm{mmHg}$, and pulse of 80 beats per minute. Except for a II/VI systolic ejection murmur at the left sternal border, the physical examination was within normal limits.

Laboratory evaluation revealed a white blood cell count of $9,200 / \mathrm{ml}$ with $17 \%$ neutrophils, $57 \%$ lymphocytes, and $26 \%$ monocytes; hematocrit was $28 \%$, and platelet count was $321,000 / \mathrm{ml}$. WESR was $114 \mathrm{~mm} /$ hour. Alkaline phosphatase was $347 \mathrm{IU} / \mathrm{L}$, aspartate aminotransferase was $78 \mathrm{IU} / \mathrm{L}$, and alanine aminotransferase $100 \mathrm{IU} / \mathrm{L}$; urinalysis showed no cells, and creatine kinase was $1366 \mathrm{U} / \mathrm{L}$. Chest roentgenogram revealed no abnormalities.

An extensive work-up revealed the following to be normal or negative: abdominal ultrasound and CT scan, cardiac echocardiogram, antinuclear antibody, rheumatoid factor, and serology for bacterial, viral, and fungal pathogens. Flow cytometry showed polyclonal $B$ cell lymphocytosis. A bone marrow biopsy revealed a few nonspecific lymphocyte aggregates; liver biopsy showed non-diagnostic increase in portal and sinusoidal lymphocytes. No cause for his fevers was found.

In April 1991, he was readmitted with intermittent fevers occurring every few weeks and lasting for several days. Again, he had elevated liver function tests, anemia, and elevated creatine kinase. He underwent an extensive work-up including a temporal artery biopsy, which was negative. Over the next few months the patient remained afebrile but had weakness, anorexia, and myalgias.

In August 1991 his fevers recurred, this time associated with diffuse abdominal pain. Bone marrow biopsy revealed a hypercellular marrow with granulocytic hyperplasia, and cultures were negative. Flow cytometric analysis of peripheral blood showed a polyclonal increase in B lymphocytes. CT, gallium, and indium scans were all within normal limits. Muscle biopsy showed no evidence of myositis. No cause for his fevers was established. In October 1991 he was begun on prednisone, $30 \mathrm{mg} /$ day. He responded with defervescence, improved appetite, resolution of symptoms, and the WESR decreased to $20 \mathrm{~mm} /$ hour.

However, 6 weeks later the patient had gained 30 pounds and developed severe back pain. When the prednisone dose was tapered, the fevers recurred, and the sedimentation rate rose again. It was noted that he had a new T6 compression fracture (Figure 3). Further studies revealed lytic skull lesions (Figure 4), uptake at T6 and T8 on bone scan, and abnormalities at T6 and T8 on MR scan (Figure 5). Needle biopsy of T8 was nonrevealing; surgical biopsy of the skull showed normal-appearing lymphocytes.

He continued to have intermittent fevers, myalgias, abdominal pain, and persistent fatigue. Finally, almost 1.5 years after the onset of his illness, flow cytometric studies of peripheral blood revealed a monoclonal population of B lymphocytes positive for CD-19, CD-20, CD-5, immunoglobulin $D$, and kappa markers. This finding, along with the skull biopsy and a third bone marrow biopsy, which showed infiltration with a homogeneous population of well differentiated small lymphocytes, was interpreted as evidence for a lowgrade B cell lymphoma. 


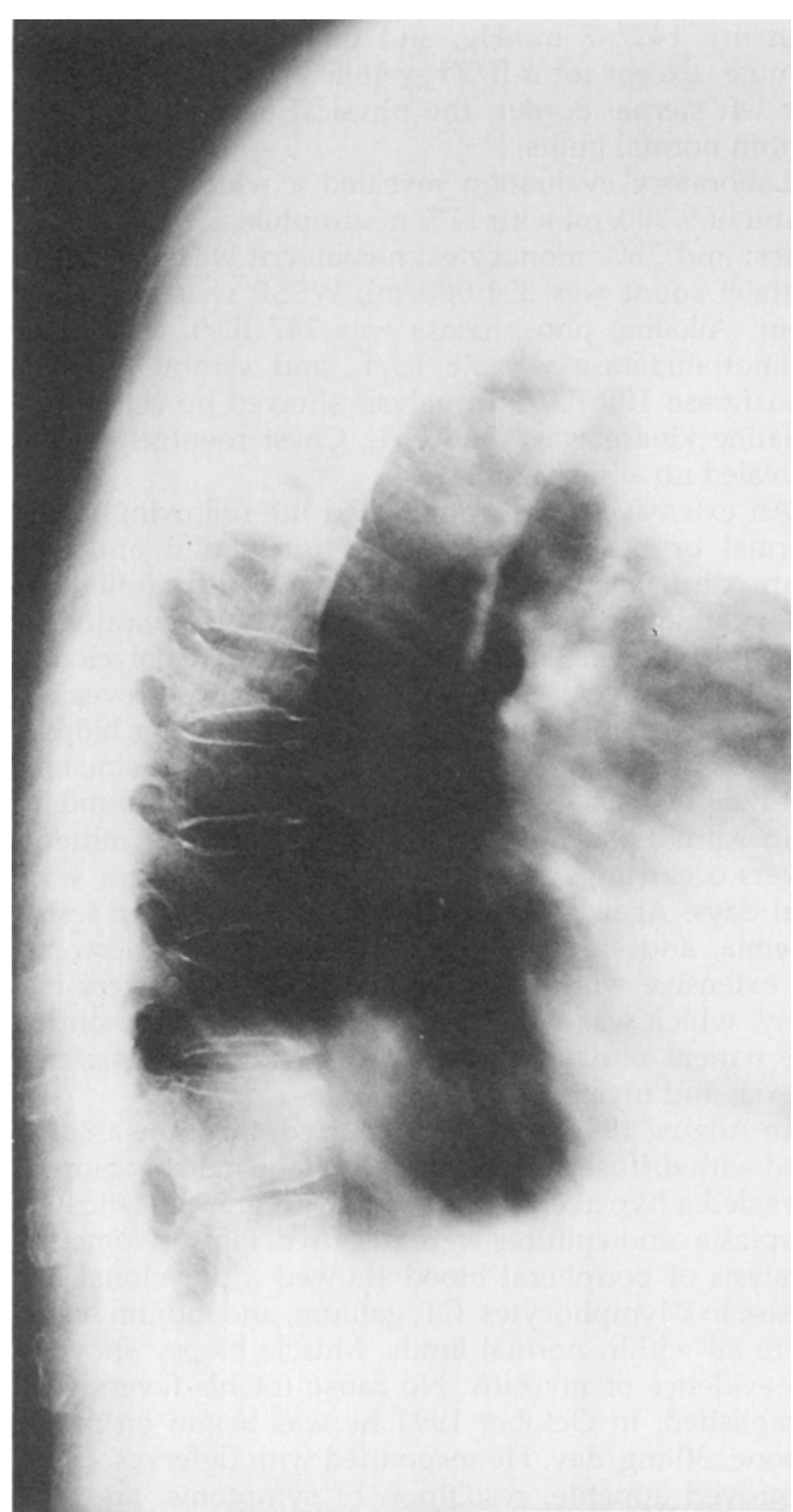

FIGURE 3. Patient 2. Lateral chest roentgenogram showing a T6 compression fracture, early changes in the T8 vertebra, and diffuse osteopenia of the spine.

\section{DISCUSSION}

The criteria for FUO as defined by Petersdorf and Beeson in their classic 1961 paper and modified by Petersdorf in 1992 are: fever higher than $101 \mathrm{~F}$ $\left(38.3^{\circ} \mathrm{C}\right)$, present for at least 3 weeks, and failure to make a diagnosis after 1 week of intelligent investigation. ${ }^{1,9}$ More than the 3 decades since their initial review, the major categories of FUO in all age groups remain infectious diseases, malignancies, and collagen vascular diseases. ${ }^{1-4}$

Although most studies of FUO in adults of all ages, as well as in the elderly, have found infections to be the most common cause of $\mathrm{FUO}^{1-6}$ neoplasms have been noted as the second most common cause. Approximately $20 \%$ to $30 \%$ of adults with FUO are found to have neoplasm in most series, ${ }^{1,2,4}$ although a recent study by Knockaert et al found that only $7 \%$ of their

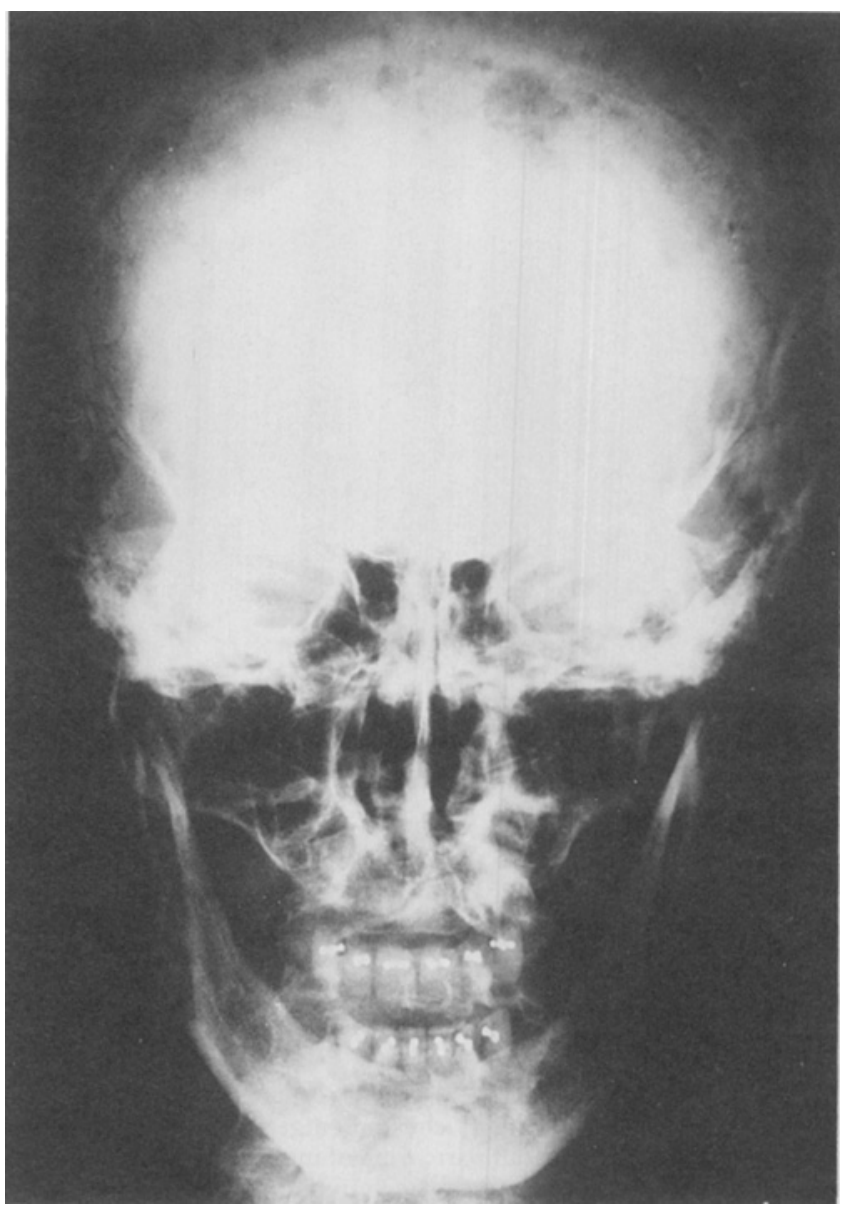

FIGURE 4. Patient 2. Multiple lytic lesions in the skull.

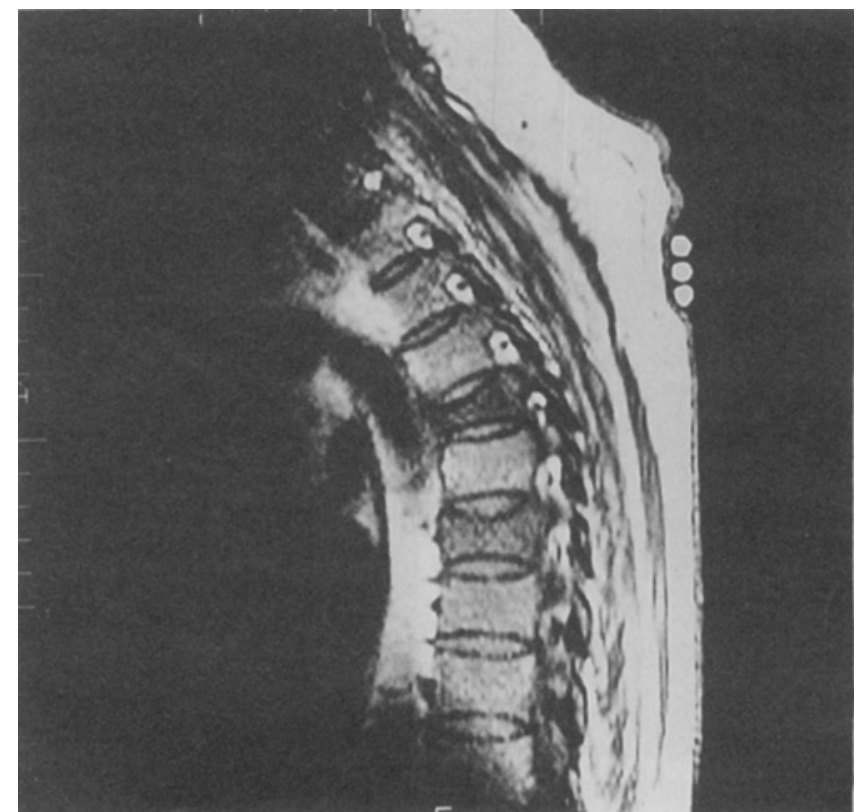

FIGURE 5. Patient 2. MR imaging study showing low-intensity signal at T6 and T8 on T2-weighted images and preservation of the intervertebral disk spaces, consistent with a neoplastic process.

patients had FUO due to neoplasms. ${ }^{3}$ Esposito and Gleckman noted that $24 \%$ of elderly patients were ultimately found to have neoplasms. ${ }^{5}$ 
Fever is a well-known manifestation of neoplastic disease, particularly disease originating in the hematopoietic system and/or metastatic to the liver. ${ }^{1,2,10,11}$ A number of different mechanisms have been postulated as causes of fevers associated with malignancies, including tumor necrosis, inflammation, and increased heat from tumor cells, ${ }^{10}$ but it appears likely that tumor-associated fever in most patients is due to the production of cytokines, such as interleukin-1, either by tumor cells or mononuclear cells. ${ }^{12-14}$

Numerous studies have found lymphoma to be the neoplasm most commonly associated with the syndrome of FUO in adults of all ages ${ }^{1,2,4}$ and in the elderly. ${ }^{5,6}$ Periodic (Pel-Ebstein) fevers have been described in association with lymphomas, usually Hodgkin's disease. ${ }^{15}$ However, these periodic fevers are rarely noted in current discussions of FUO, perhaps because patients are often treated with antipyretic agents, which change fever patterns. The second patient described had periodic fevers. Although the periodicity was longer than that described in most cases, in which the fever usually recurs every several weeks, the pattern of weeks to months of afebrility interrupted by weeks of daily temperature elevation was similar to that described as Pel-Ebstein fever. ${ }^{15}$

Both of our patients had vertebral involvement with lymphoma leading to vertebral collapse. The first patient progressed to spinal cord compression in less than 2 months. The second patient progressed more slowly; vertebral collapse occurred after 1 year of illness, and cord compression never developed. Vertebral involvement with lymphoma is not common. ${ }^{16,17}$ Even when cord compression is present, there may be no changes noted on roentgenogram of the spine. ${ }^{16,17}$

In the elderly, the usual presumption is that back pain and vertebral compression fractures are the consequences of aging. That assumption is not unreasonable given that osteoporosis is common in the elderly. ${ }^{18,19}$ However, although most vertebral fractures are probably due to involutional or primary osteoporosis, secondary causes always must be considered in the setting of FUO. Vertebral compression fractures in an elderly patient with FUO might be important clues to the diagnosis of multiple myeloma, lymphoma, leukemia, or metastatic neoplasms, which stimulate an increase in bone resorption. ${ }^{18}$ Likewise, thyroid hormone excess states can cause both FUO and compression fractures due to increased bone resorption. Several drugs that are associated with secondary osteoporosis (phenytoin and heparin) may also cause drug fever. Although rarely a cause of FUO, the patient who acquires secondary osteoporosis due to suppression of bone metabolism by chronic glucocorticoid or ethanol use may become febrile if the drug is withdrawn abruptly. ${ }^{18,19}$

Vertebral osteomyelitis should always be considered in elderly patients with back pain, vertebral compression fractures, and fever. ${ }^{20-22}$ Unlike compression fractures due to osteoporosis, which predominate in females, vertebral osteomyelitis occurs more often in elderly men. ${ }^{20}$ McHenry et al found that in the elderly osteoporosis accounted for $67 \%$ of vertebral fractures, whereas metastatic tumors (14\%), myeloma $(3 \%)$, osteomyelitis $(2 \%)$, and lymphoma $(1 \%)$ were relatively rare. ${ }^{21}$ Osteomyelitis is distinguished from other causes of vertebral compression fracture by its propensity to involve the disk space. ${ }^{2,23}$

Roentgenograms may be difficult to interpret unless marked erosion of the disk space suggesting infection is present. ${ }^{21-24}$ These changes often lag behind those noted on nuclear or CT scans. ${ }^{22-24}$ Gallium and technetium bone scans are very sensitive but cannot distinguish between infection, tumor, fracture, or rheumatologic diseases. ${ }^{21-23} \mathrm{CT}$ scan is helpful in identifying early cortical erosions not seen on roentgenograms, as well as delineating soft tissue swelling. However, assessment of disk space involvement, the most important diagnostic clue in the diagnosis of infection, is best observed using MR imaging. ${ }^{22,23} \mathrm{MR}$ is probably the procedure of choice in the elderly patient who has FUO and compression fractures and who might have either osteomyelitis or neoplasm. ${ }^{23}$ If the disk space is normal and the body of the vertebra only is involved, then neoplasm is much more likely than infection.

Our patients illustrate the axiom that most cases of FUO are due to atypical presentations of common diseases. ${ }^{2}$ The use of diagnostic procedures and laboratory tests in the diagnosis of FUO should be guided by a careful assessment of the patient's history, findings on physical examination, and preliminary laboratory results. Complaints of back pain and the finding of compression fractures should not be ascribed to osteoporosis related to aging in the febrile elderly patient, but should be aggressively pursued with regard to possible infectious or neoplastic causes.

\section{REFERENCES}

1. Petersdorf RG, Beeson PB. Fever of unexplained origin: Report of 100 cases. Medicine 1961;40:1-30.

2. Larson EB, Featherstone HJ, Petersdorf RG. Fever of undetermined origin: Diagnosis and follow-up of 105 cases, 1970-1980. Medicine 1982;61: 269-293.

3. Knockaert DC, Vanneste LJ, Vanneste SB, Bobbaers HJ. Fever of unknown origin in the 1980's: An update of the diagnostic spectrum. Arch Intern Med 1992;152:51-55.

4. Kazanjian PH. Fever of unknown origin: Review of 86 patients treated in community hospitals. Clin Infect Dis 1992;15:968-973.

5. Esposito AL, Gleckman R. Fever of unknown origin in the elderly. J Am Geriatr Soc 1978;26:498-505.

6. Kauffman CA, Jones PG. Diagnosing fever of unknown origin in older patients. Geriatrics 1984;39:46-51.

7. Rumans LW, Vosti KL. Factitious and fraudulent fever. Am J Med 1978;65:745-755.

8. Marcus E-L, van Dijk JM. Fever of unknown origin. J Am Geriatr Soc 1991;39:637-638.

9. Petersdorf RG. Fever of unknown origin. An old friend revisited. Arch Intern Med 1992;152:21-22.

10. Chang JC. Neoplastic fever: A proposal for diagnosis. Arch Intern Med 1989;149:1728-1730.

11. Bodel P. Tumors and fever. Ann NY Acad Sci 1974;230:6-13.

12. Bodel $P$, Ralph $P$, Wenc $K$, Long JC. Endogenous pyrogen production by Hodgkin's disease and human histiocytic lymphoma cell lines in vitro. $J$ Clin Invest 1980;65:514-518.

13. Dinarello CA. Interleukin-1. Rev Infect Dis 1984;6:51-95.

14. Lachman LB, Moore JO, Metzger RS. Preparation and characterization of LAF from acute monocytic and myelomonocytic leukemic cells. Cell Immunol 1978;41:189-206.

15. Reimann HA. Periodic (Pel-Ebstein) fever of lymphomas. Ann Clin Lab Sci 1977;7:1-5.

16. Mullins GM, Flynn JPG, El-Mahdi AM, McQueen JD, Owens AH. Malignant lymphoma of the spinal epidural space. Ann Intern Med $1971 ; 74: 416-423$.

17. Fornasier VL, Horne JG. Metastases to the vertebral column. Cancer 1975;36:590-594. 
18. Jackson JA, Kleerekoper M. Osteoporosis in men: Diagnosis, pathophysiology, and prevention. Medicine 1990;69:137-152

19. Rubin CD. Southwestern Internal Medicine Conference: Age-related osteoporosis. Am J Med Sci 1991;301:281-298.

20. Sapico FL, Montgomerie JZ. Pyogenic vertebral osteomyelitis: Report of nine cases and review of the literature. Rev Infect Dis 1979;1:754-776.

21. McHenry MC, Duchesneau PM, Keys TF, Rehm SJ, Boumphrey FRS Vertebral osteomyelitis presenting as spinal compression fracture: Six patients with underlying osteoporosis. Arch Intern Med 1988;148 417-423.

22. Cahill DW, Love LC, Rechtine GR. Pyogenic osteomyelitis of the spine in the elderly. J Neurosurg 1991;74:878-886.

23. Smith AS, Blaser SI. Infectious and inflammatory processes of the spine. Radiol Clin North Am 1991;29:809-827.

24. Ansari A, Lundquist VP, Azad M. Bone scintiscan and malignant lymphoma. Ann Intern Med 1976;85:684-685. 